\title{
Ciclo de mejora en el aula para la asignatura Teoría de la argumentación.
}

\section{Cycle of Improvement in the Classroom for an Argumentation Theory's course}

CRISTINA BARÉS GÓMEZ

Universidad de Sevilla. Departamento de

Filosofía, Lógica y Filosofía de la ciencia

crisbares@gmail.com

ORCID: https://orcid.org/0000-0002-8132-9320

DOI: http://dx.doi.org/10.12795/9788447231003.002

Pp.: 51-66 


\section{Breve descripción del contexto}

La asignatura para la que se realiza este Ciclo de Mejora en el Aula (CIMA) es Teoría de la argumentación. Es una asignatura obligatoria de 60 horas del Grado de Filosofía y el doble Grado Filosofía y Derecho de la Universidad de Sevilla, primer año. El curso en el que se implementó, fue un curso bajo restricciones COVID-19 por lo que nos enfrentamos a una situación de docencia híbrida organizada mediante un 33\% (3 grupos). Cada grupo de alumnos viene a clase durante una semana y dos semanas online. Las clases transcurren con un grupo en presencial y dos grupos online con un total de 53 alumnos.

El contenido de la asignatura es un contenido básico, pues es la primera vez que los estudiantes se enfrentan a las asignaturas del área. La mayoría de los alumnos no tienen nociones de lógica y tampoco de teoría de la argumentación. Sólo algunos han visto algunas nociones de lógica proposicional en el bachillerato. Por ello nuestro punto de partida suele ser de 0 , ello nos permite ir construyendo paulatinamente la estructura de la asignatura desde las bases.

A continuación expondré los diferentes apartados desarrollados en el ciclo de mejora empezando por un mapa conceptual en el que tendré en cuenta no solo los aspectos conceptuales, sino también los procedimentales y actitudinales. Seguidamente, explicaré en detalle la organización del ciclo de mejora previa a su realización y el cuestionario inicial y final realizado. Posteriormente, haré una evaluación detallada de algunas preguntas del cuestionario y del ciclo de mejora. La última parte está dedicada a detallar los principios didácticos en los que me he basado.

Ciclos de Mejora en el Aula (2020). Experiencias de Innovación Docente de la US Esta obra se distribuye con la licencia Creative Commons 


\section{Mapa de conceptual}

Para un primer desarrollo del ciclo de mejora, es necesario estructurar los contenidos del ciclo. A continuación se exponen los contenidos que se van a tener en cuenta en la realización del cuestionario y el desarrollo del CIMA. No son solo contenidos conceptuales, pues la finalidad última no es solo la memorización de los conceptos, sino que se pretende dotar de herramientas a los estudiantes para poder analizar el discurso y los argumentos. Por ello, no solo son fundamentales los contenidos procedimentales, sino también los actitudinales más allá de la mera sucesión de conceptos.

Conceptos:

- Noción de significado. Sintaxis y semántica. Distintas semánticas.

- Nociones de verdad. Verdad como correspondencia Verdad como construcción.

- Noción de consecuencia lógica. Esquema de argumento - necesidad y propiedades formales.

Procedimientos.

- Entender y ser capaz de reconocer los fundamentos de la noción de significado y semántica que usamos a la hora de evaluar los argumentos.

- Saber cómo se evalúa un argumento o esquema de argumento, en función de qué parámetros.

- Diferenciar corrección de un argumento y verdad de una proposición.

- Conocer y reconocer la noción de consecuencia lógica y ser conscientes cuando se cumple y cuando no.

- Reconocer las implicaciones del no cumplimiento de la corrección de los argumentos.

- Ser capaz de reconocer la noción de verdad que usamos y sus consecuencias. Compromisos de los agentes.

Ciclos de Mejora en el Aula (2020). Experiencias de Innovación Docente de la US Esta obra se distribuye con la licencia Creative Commons 
Actitudes

- Honestidad intelectual en el uso de las herramientas argumentativas.

- Ser capaz de argumentar correctamente, en el sentido de ser consciente de los compromisos que llevan las proposiciones que afirmamos.

- Normalmente, cuando se argumenta, se intenta hacer desde un punto de vista racional para aquellos que consideramos personas racionales. Aunque no siempre se cumple.

- Si no argumentamos correctamente, ser conscientes de lo que hacemos.

La base conceptual de toda la asignatura es el esquema de argumento.

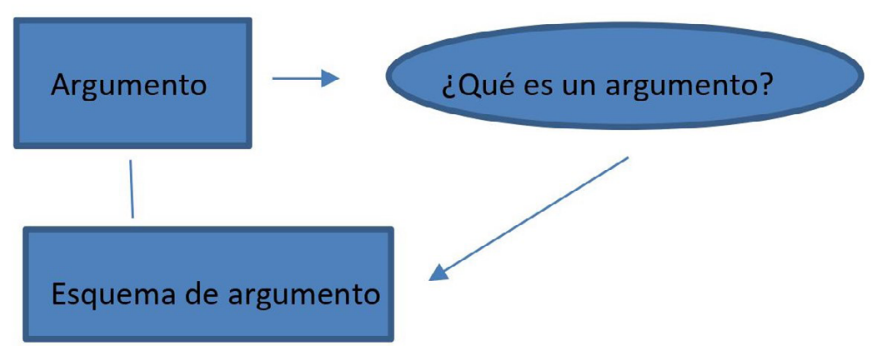

Relación

Premisas

Conclusión

Figura 1. Esquema de argumento

Ciclos de Mejora en el Aula (2020). Experiencias de Innovación Docente de la US Esta obra se distribuye con la licencia Creative Commons 
Relación

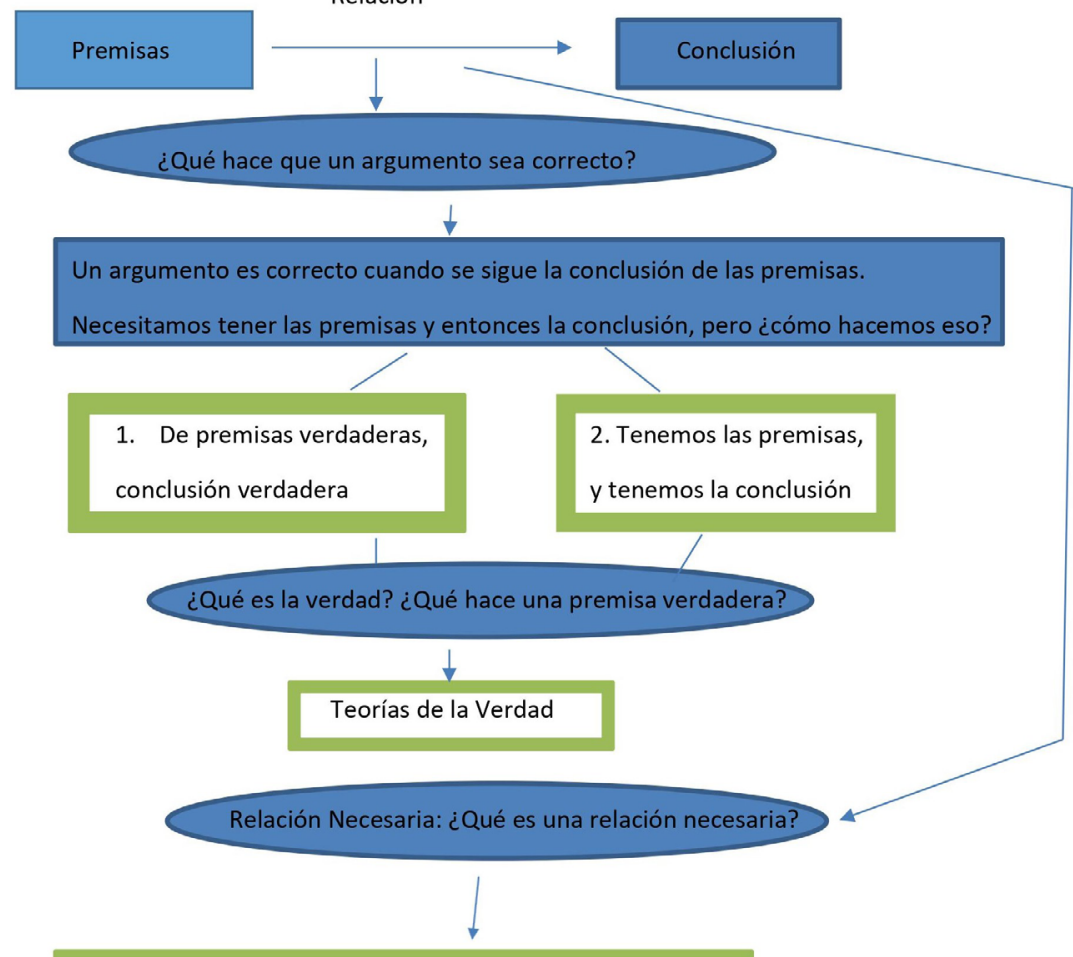

Relación de consecuencia lógica. Propiedades formales

Figura 2. Relación de los temas tratados en este CIMA con el esquema de argumento

\section{Organización}

Una vez establecidos los contenidos, procedemos a establecer una organización previa de las sesiones. Esta planificación, puede ser, de hecho fue, modificada en la realización, pues al final me fui adaptando al ritmo de la clase. El CIMA se organiza en cuatro sesiones de dos horas cada una siguiendo la siguiente estructura previa:

Sesión I. 2 horas. 23 octubre 2020

- Cuestionario. 20 min.

- Base para la actividad: Deja que hablen los libros. Finkel, D. (2008) - 45 min.

Ciclos de Mejora en el Aula (2020). Experiencias de Innovación Docente de la US Esta obra se distribuye con la licencia Creative Commons 
- Texto: Enunciado. Vega, Luis y Olmos, Paula. (ed.). (2011) $-20 \mathrm{~min}$.

- Puesta en común en grupo y puesta en común general por un representante de cada grupo. Actividad de contraste - 25 min.

- Pausa 10 min.

- Base para la actividad: deja que hablen los libros. Finkel, D. (2008) - 45 min.

- Significado - Saussure - signo. - Vega, Luis y Olmos, Paula. (ed.). (2011) - $20 \mathrm{~min}$.

- Puesta en común en grupo y puesta en común general por un representante de cada grupo. Actividad de contraste $25 \mathrm{~min}$.

Sesión II - 2 horas. 29 octubre 2020. Base para la actividad: Taller conceptual, Finkel, D. (2008). Texto: Sentido y referencia. Frege, G. (1991).

- Lectura individual primera parte y puesta en común en grupo - 20 min.

- Puesta en común general y actividad de contraste - 25 min.

- Pausa 10 min.

- Lectura individual segunda parte y puesta en común en grupo - 20 min.

- Puesta en común general y actividad de contraste - 25 min.

Sesión III - 2 horas. 30 octubre 2020. Base para la actividad: Taller conceptual, Finkel, D. (2008). Texto: Wittgenstein a propósito de reglas y lenguaje privado. Kripke, S. (2006).

- Lectura individual - 20 min.

- Puesta en común en grupo 25 min.

- Pausa 10 min.

- Puesta en común general 10 min.

- Actividad de contraste - solución - 35 min.

Ciclos de Mejora en el Aula (2020). Experiencias de Innovación Docente de la US Esta obra se distribuye con la licencia Creative Commons 
Sesión IV- 2 horas. 5 noviembre 2020. Base para la actividad: Taller conceptual, Finkel, D. (2008). Texto: Thinking about Logic. An Introduction to the Philosophy of Logic. Read, S. (1995). Puesta en práctica de sesión II y III en los argumentos.

- Lectura individual y comentario grupal - 25 min.

- Pausa 10 min.

- Actividad de Contraste final. Teorías de la verdad y consecuencia lógica. $45 \mathrm{~min}$.

- Cuestionario final. $20 \mathrm{~min}$.

\section{Cuestionario inicial-final}

Antes de la realización del CIMA, en la primera sesión como se ha indicado en la organización, hice un cuestionario a todos los estudiantes. La finalidad del cuestionario es conocer los modelos mentales y la situación inicial de los estudiantes respecto a la asignatura. Este cuestionario se pasé de nuevo al final de las sesiones del ciclo de mejora para ver la evolución de los alumnos. El cuestionario utilizado fue el siguiente:

Instrucciones: Esta encuesta es meramente orientativa y no entra en la evaluación del curso. No ponga su nombre, sino un alias, color, nombre ficticio, número, etc. Sólo quiero que lo recuerde para poner el mismo en el cuestionario final.

Alias:

Pregunta 1. ¿Qué es el significado? Responde brevemente y da dos ejemplos de elementos y su significado.

Pregunta 2. Explica la semántica de la siguiente proposición: Llueve en Sevilla.

Pregunta 3. Pon un ejemplo de una conclusión y unas premisas en las que siempre que tengamos las premisas, tendremos la conclusión. Después dime dónde está el significado de este esquema de argumento.

Ciclos de Mejora en el Aula (2020). Experiencias de Innovación Docente de la US Esta obra se distribuye con la licencia Creative Commons 
Pregunta 4. ¿Qué hace que un elemento sea idéntico a otro? Dame un ejemplo de cosas idénticas.

Pregunta 5. ¿Explica qué necesita un jugador para jugar a un juego cualquiera? Dirige tu explicación con un ejemplo de juego.

Pregunta 6. ¿Qué es la verdad? Pon un ejemplo de un elemento verdadero.

Pregunta 7. Cuando decimos que la conclusión se sigue necesariamente de las premisas, ¿a qué se refiere ese "se sigue"? Dame alguna de las características.

\section{Aplicación del CIMA}

Como ya hemos indicado, la implementación del segundo CIMA de 8 horas se dividió en cuatro sesiones de 2 horas cada una. A continuación se detalla su aplicación que no coincide exactamente con la organización planificada en un principio. Haré un análisis de cada una de ellas por separado y luego en conjunto.

Sesión I: Estaba dedicada al cuestionario inicial y al trabajo de dos textos. Los textos propuestos fueron los textos relativos al enunciado y al signo según Saussure. La estrategia didáctica fue dejar que los textos hablarán y ponerlos seguidamente en común. Debido a problemas técnicos por la situación COVID-19 perdimos tiempo con la plataforma. Después, al ser la primera vez que hacía una clase por grupos, tardamos más en organizarnos. La dinámica fue la siguiente, dejar tiempo para que los estudiantes leyeran el primer texto; hacer tres grupos según la organización COVID-19 del 33\% (uno en clase y dos online) y comentar el texto; nombrar a un representante de cada grupo para que pusiera en común las conclusiones de su grupo en una lluvia de ideas en la pizarra. Tras la puesta en común, yo retomé las explicaciones y fui aclarando todo lo que había salido en la pizarra. El curso no fue mal, de hecho los estudiantes se implicaron bastante, pero me faltó

Ciclos de Mejora en el Aula (2020). Experiencias de Innovación Docente de la US Esta obra se distribuye con la licencia Creative Commons 
tiempo y solo pude trabajar con ellos uno de los dos textos propuestos.

Sesión II. El texto propuesto fue el texto Sentido y Referencia de Frege. En lugar de darles el texto directamente, realizamos un taller conceptual con varios bloques siguiendo la misma estructura. Primero leían las preguntas y contestaban individualmente, luego en grupos y más tarde en común, retomando todo al final como actividad de contraste. Igualmente fue fructífero, pero también se hizo demasiado largo y no me dio tiempo a terminar el taller. Después del taller, les di el texto para que lo siguieran trabajando en casa.

Sesión III. El taller conceptual fue sobre el texto de Kripke, Wittgenstein: sobre reglas y lenguajes privados. Les plantee la paradoja de los lenguajes privados con varias preguntas y dejé tiempo para trabajarla primero individualmente y luego por grupos. En este caso la organización del tiempo fue acertada, incluso me sobraron unos minutos para varias dudas. Lo que percibí en esta ocasión es que no se fueron muchos a los grupos y lo que querían era discutir todos en el mismo grupo.

Sesión IV. La realización del taller conceptual tuvo la misma organización y parece que conseguí que fuera provechoso. El tiempo se cuadró bien, pero me faltó tiempo para el cuestionario final y algunos lo hicieron más tarde online.

En general, creo que el segundo CIMA ha sido bueno. Tal vez reduciría un poco el tiempo de los grupos y pondría más en un grupo general. Supongo que en otras condiciones que no sean de docencia híbrida puede funcionar mejor.

\section{Evaluación del cuestionario}

Los cuestionarios fueron de dos tipos, presenciales (cuestionario en papel) y online (encuestas EV-US). En ambos casos fueron anónimos y se emparejaron el inicial y el

Ciclos de Mejora en el Aula (2020). Experiencias de Innovación Docente de la US Esta obra se distribuye con la licencia Creative Commons 
final por el alias. Ello produjo que los cuestionarios que no tenían el mismo alias no pudieron entrar en la evaluación. Para la realización de la evaluación nos hemos basado en el capítulo 4 "La evaluación en la enseñanza universitaria" de Rivero, A y Porlán, R. en Porlan, R. (coord.)(2017).

Pregunta 2: Explica la semántica de la siguiente proposición: Llueve en Sevilla.

Tabla 1. Escalera pregunta 2, general

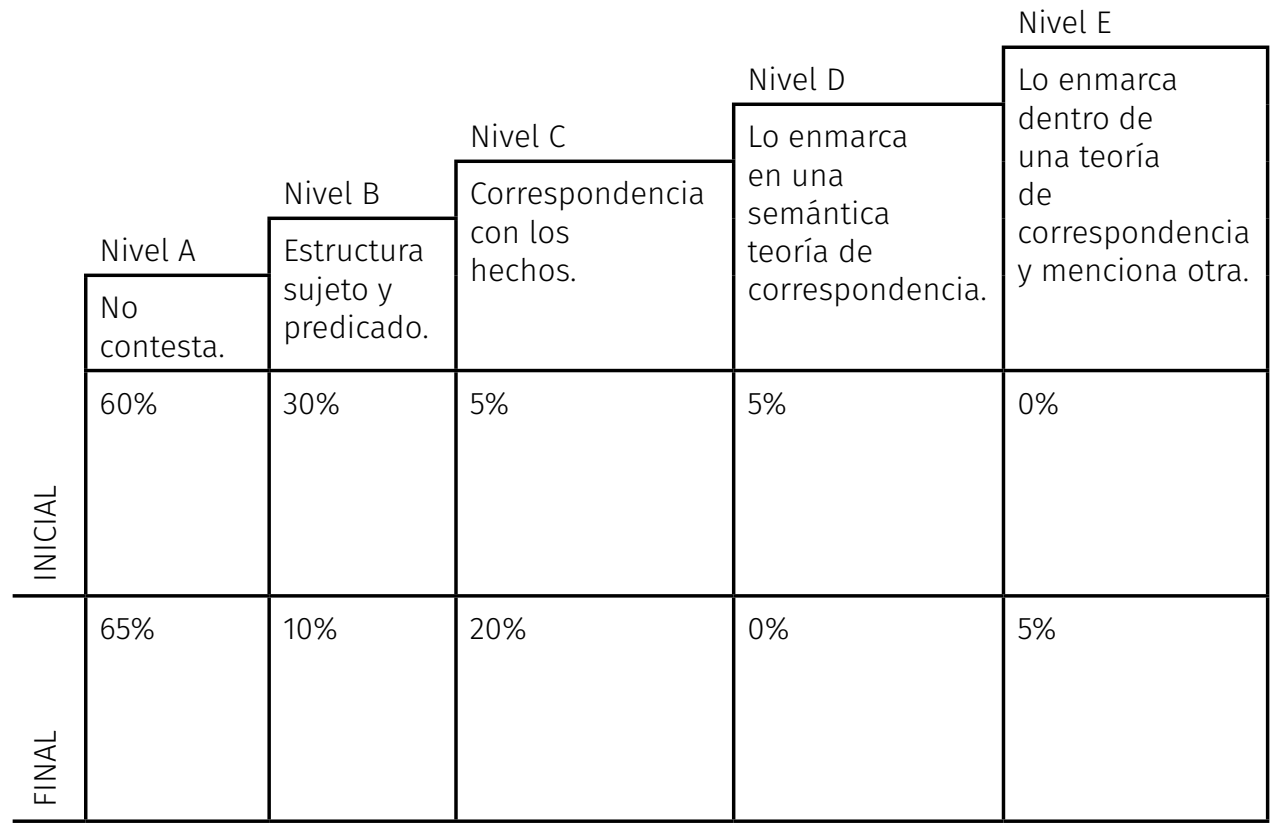

Ciclos de Mejora en el Aula (2020). Experiencias de Innovación Docente de la US Esta obra se distribuye con la licencia Creative Commons 
Tabla 2. Evaluación pregunta 2 por alumnos

\begin{tabular}{|l|l|l|l|}
\hline & Inicial & Final & Avance \\
\hline Alumno 1 & D & E & $\uparrow$ \\
\hline Alumno 2 & A & A & $\leftrightarrow$ \\
\hline Alumno 3 & A & B & $\uparrow$ \\
\hline Alumno 4 & C & A & $\downarrow \downarrow$ \\
\hline Alumno 5 & A & C & $\uparrow \uparrow$ \\
\hline Alumno 6 & B & A & $\downarrow$ \\
\hline Alumno 7 & A & C & $\uparrow \uparrow$ \\
\hline Alumno 8 & A & A & $\leftrightarrow$ \\
\hline Alumno 9 & A & A & $\leftrightarrow$ \\
\hline Alumno 10 & B & C & $\uparrow$ \\
\hline Alumno11 & A & B & $\uparrow$ \\
\hline Alumno12 & B & A & $\downarrow$ \\
\hline Alumno 13 & A & A & $\leftrightarrow$ \\
\hline Alumno 14 & B & A & $\downarrow$ \\
\hline Alumno15 & A & C & $\uparrow \uparrow$ \\
\hline Alumno16 & B & A & $\downarrow$ \\
\hline Alumno 17 & A & A & $\leftrightarrow$ \\
\hline Alumno 18 & A & A & $\leftrightarrow$ \\
\hline Alumno 19 & B & A & $\downarrow$ \\
\hline Alumno 20 & A & A & $\leftrightarrow$ \\
\hline
\end{tabular}

Comentario. Podemos observar que hay algunos alumnos que han subido en la escalera, pero hay muchos que se han mantenido en el primer nivel. Lo que resulta más curioso es la cantidad de alumnos que han bajado en la escalera. Resulta que las respuestas muchas veces parecían sacadas de manual y en algunos casos en el cuestionario final o no han contestado o han dicho directamente que no sabían. Tal vez una posible interpretación es que se empiezan a cuestionar realmente lo que se les pregunta, pero la verdad es que no lo tengo muy claro.

Ciclos de Mejora en el Aula (2020). Experiencias de Innovación Docente de la US Esta obra se distribuye con la licencia Creative Commons 
Pregunta 8: Cuando decimos que la conclusión se sigue necesariamente de las premisas, ¿a qué se refiere ese "se sigue"? Dame alguna de las características.

Tabla 3. Escalera pregunta 8, general

\begin{tabular}{|c|c|c|c|c|c|}
\hline & & & & & Nivel E \\
\hline & & \multirow[b]{3}{*}{ Nivel B } & \multirow[b]{2}{*}{ Nivel C } & Nivel D & \multirow{5}{*}{$\begin{array}{l}\text { Menciona } \\
\text { además } \\
\text { alguna } \\
\text { propiedad } \\
\text { como por } \\
\text { ejemplo la } \\
\text { monotonía }\end{array}$} \\
\hline & & & & \multirow{4}{*}{$\begin{array}{l}\text { Además } \\
\text { reconoce } \\
\text { la deducción } \\
\text { o menciona la } \\
\text { noción de } \\
\text { consecuencia } \\
\text { lógica }\end{array}$} & \\
\hline & & & \multirow{3}{*}{$\begin{array}{l}\text { Usa una } \\
\text { definición } \\
\text { semántica de } \\
\text { preservación } \\
\text { de la } \\
\text { verdad. }\end{array}$} & & \\
\hline & Nivel A & \multirow{2}{*}{$\begin{array}{l}\text { Introduce } \\
\text { alguna } \\
\text { noción } \\
\text { semántica } \\
\text { sin acertar. }\end{array}$} & & & \\
\hline & Necesariedad & & & & \\
\hline & $95 \%$ & $5 \%$ & $0 \%$ & $0 \%$ & $0 \%$ \\
\hline & $45 \%$ & $15 \%$ & $25 \%$ & $10 \%$ & $5 \%$ \\
\hline & & & & & \\
\hline
\end{tabular}

Tabla 4. Evaluación pregunta 8 por alumnos

\begin{tabular}{|l|l|l|l|}
\hline & Inicial & Final & Avance \\
\hline Alumno 1 & A & A & $\leftrightarrow$ \\
\hline Alumno 2 & A & A & $\leftrightarrow$ \\
\hline Alumno 3 & A & B & $\uparrow$ \\
\hline Alumno 4 & A & D & $\uparrow \uparrow \uparrow$ \\
\hline Alumno 5 & A & C & $\uparrow \uparrow$ \\
\hline Alumno 6 & A & C & $\uparrow \uparrow$ \\
\hline Alumno 7 & A & A & $\leftrightarrow$ \\
\hline Alumno 8 & A & A & $\leftrightarrow$ \\
\hline Alumno 9 & A & B & $\uparrow$ \\
\hline
\end{tabular}

Ciclos de Mejora en el Aula (2020). Experiencias de Innovación Docente de la US Esta obra se distribuye con la licencia Creative Commons 


\begin{tabular}{|l|l|l|l|}
\hline Alumno 10 & B & B & $\leftrightarrow$ \\
\hline Alumno11 & A & A & $\leftrightarrow$ \\
\hline Alumno12 & A & E & $\uparrow \uparrow \uparrow \uparrow$ \\
\hline Alumno 13 & A & D & $\uparrow \uparrow \uparrow$ \\
\hline Alumno 14 & A & C & $\uparrow \uparrow$ \\
\hline Alumno15 & A & A & $\leftrightarrow$ \\
\hline Alumno16 & A & C & $\uparrow \uparrow \uparrow$ \\
\hline Alumno 17 & A & A & $\leftrightarrow$ \\
\hline Alumno 18 & A & A & $\leftrightarrow$ \\
\hline Alumno 19 & A & A & $\leftrightarrow$ \\
\hline Alumno 20 & A & C & $\uparrow \uparrow \uparrow$ \\
\hline
\end{tabular}

Comentario. Podemos observar como la mitad de estudiantes han subido en la escala. Algunos incluso han subido cuatro escalones.

\section{Evaluación general CIMA}

El aprendizaje de los alumnos lo he analizado por medio del cuestionario, inicial y final, y a través de sus intervenciones en clase y en tutorías. Creo que algunos, no todos, han conseguido al menos llegar a la base fundamental de la estructura de la asignatura, pero desde mi punto de vista, todavía están lejos de mis objetivos. El hecho de centrarme en sus modelos mentales y su perspectiva, tal vez haya facilitado el movimiento de los principios establecidos y no reflexionados de varias estructuras conceptuales. Creo que más bien he hecho que empiecen a plantearse cuestiones que antes no se planteaban y que no estudien simplemente para memorizar la asignatura. Pero de ahí a que tengan una estructura clara de la asignatura y los conceptos creo que todavía tengo un largo camino que recorrer.

He procurado seguir la asignatura con los contenidos que ya había diseñado y centrarla más en un hilo común.

Ciclos de Mejora en el Aula (2020). Experiencias de Innovación Docente de la US Esta obra se distribuye con la licencia Creative Commons 
El centro es el esquema de argumento y he intentado hacer toda la presentación del curso en torno a este concepto. Los contenidos por así decirlo, no han cambiado mucho, pues yo misma los había diseñado anteriormente. Lo que he hecho es perfeccionar la estructura y las relaciones para que queden más claras para los alumnos cambiando algunos textos. Eso sí, creo que algunos contenidos no cuadran mucho con toda la estructura, no los que he usado en el CIMA, sino antes y tal vez los modifique la próxima vez. Para la próxima vez se me ha ocurrido añadir otros contenidos más cercanos a los estudiantes que debo trabajar.

Las secuencias de actividades han ido bien. Mi mayor problema ha sido el tiempo. En las dos primeras sesiones había preparado demasiado material y no pude terminarlo. Creo que tengo que centrarme mejor en lo esencial e intentar organizarme un poco mejor para hacerlo. He cambiado dos textos en el temario para dos talleres conceptuales y creo que eso sí ha sido un acierto. El hecho de darles tiempo para que se planteen los problemas antes, creo que también ha sido fructífero en el sentido de que les he acercado los conceptos y ahora ellos mismo se hacen las preguntas.

La evaluación, excepto en unos dos o tres casos me ha sorprendido y en un principio no sé si debía tomármelo como algo positivo o negativo. Hay muchos estudiantes que han bajado de nivel en la escalera conceptual. En el cuestionario inicial me he encontrado con multitud de respuestas tipo sacadas de manual, mientras que en el segundo me he encontrado con muchas preguntas sin respuesta o con frases literalmente "No lo sé". Me gustaría pensar que es porque realmente estoy cambiando los modelos mentales y no solo las respuestas tipo que están habituados a dar en los cuestionarios. De hecho, no creo que ningún estudiante haya llegado al nivel más alto de las escaleras en todas las preguntas. Lo que me ha resultado útil

Ciclos de Mejora en el Aula (2020). Experiencias de Innovación Docente de la US Esta obra se distribuye con la licencia Creative Commons 
es ver los errores conceptuales que cometen y reconocer los aspectos en los que tengo que insistir.

Principios didácticos de base para el CIMA

Los principios didácticos en los que me he basado para la realización de este CIMA se fundamentan en una mayor relación de intercambio entre el profesor y el alumno. He intentado primero conocer el punto de partida de los estudiantes respecto a la asignatura e ir construyendo las sesiones en función de sus necesidades. Con las actividades "deja que hablen los textos" he promovido una primera aproximación individual a los conceptos que luego se han puesto en común con los iguales en grupo y se han complementado con las actividades de contraste. Ello pretende que los alumnos trabajen desde sus puntos de vista y no actúen como meros receptores del conocimiento. Las actividades de "taller conceptual" tienen una finalidad similar. De hecho, la intención de estos talleres es hacer pensar a los estudiantes para que busquen respuestas a partir de problemas o preguntas. He expuesto problemas fundamentales para la teoría del significado tales como el problema de la identidad en Frege, G (1991) y el problema o la paradoja de Wittgenstein en Kripke, S. (2006). Pretendía que los estudiantes entendieran primero el problema para trabajar en la respuesta posteriormente. Creo que es fundamental que los alumnos entiendan los problemas y sus implicaciones para que los conceptos no sean una mera sucesión memorística de teorías. Por último, en el taller sobre las teorías de la verdad y la consecuencia lógica, pretendía que tuvieran las herramientas suficientes para que fueran capaces de analizar la corrección de un argumento y reconocer los principios en los que nos basamos para establecerla.

Ciclos de Mejora en el Aula (2020). Experiencias de Innovación Docente de la US Esta obra se distribuye con la licencia Creative Commons 
Palabras clave: Teoría de la argumentación, Semántica, Teorías de la verdad, Consecuencia lógica, docencia universitaria, experimentación docente.

Key word: Argumentation Theory, Semantics, Theories of Truth, Logical Consequence, university teaching, university teaching experimentation.

\section{Referencias bibliográficas}

Finkel, D. (2008). Dar clase con la boca cerrada. (O. Barberá, trad.). Valencia: Servicio de Publicaciones de la Universidad de Valencia.

Frege, G. (1991). Sobre sentido y Referencia. (L. Valdés, trad.). En La búsqueda del significado. Madrid: Tecnos. (original publicado en 1879)

Kripke, S. (2006). Wittgenstein a propósito de reglas y lenguaje privado. (J. Rodríguez Marqueze, trad.). Madrid: Tecnos. (original publicado en 1982)

Porlan, R. (Coord.) (2017). Enseñanza universitaria. Cómo mejorarla. Madrid: Ed. Morata.

Read, S. (1995). Thinking about Logic. An Introduction to the Philosophy of Logic. London: Oxford University Press.

Vega, Luis y Olmos, Paula. (ed.). (2011). Compendio de Lógica Argumentación y Retórica. Madrid: Trotta.

Ciclos de Mejora en el Aula (2020). Experiencias de Innovación Docente de la US Esta obra se distribuye con la licencia Creative Commons 